\title{
Hallmark of Perfect Graphene
}

\author{
Elizabeth J. Duplock, ${ }^{1}$ Matthias Scheffler, ${ }^{2}$ and Philip J. D. Lindan ${ }^{1, *}$ \\ ${ }^{1}$ School of Physical Sciences, University of Kent, Canterbury CT2 7NR, United Kingdom \\ ${ }^{2}$ Fritz-Haber-Institut der Max-Planck-Gesellschaft, Faradayweg 4-6, D-14 195 Berlin-Dahlem, Germany
}

(Received 21 November 2003; published 3 June 2004)

\begin{abstract}
Using first-principles calculations we show that the adsorption of atomic hydrogen on graphene opens a substantial gap in the electronic density of states in which lies a spin-polarized gap state. This spin is quenched by the presence of a rotated $\mathrm{C}-\mathrm{C}$ bond (a Stone-Wales defect) adjacent to or distant from the $\mathrm{H}$ atom. We explain these findings and discuss the implications for nanotubes and magnetic nanographene. Furthermore, we demonstrate that the combined effect of high curvature and a Stone-Wales defect makes $\mathrm{H}_{2}$ chemisorption close to being thermodynamically favorable.
\end{abstract}

Carbon is unique in possessing allotropes of each possible dimensionality, from 3D diamond to $0 \mathrm{D}$ fullerenes [1]. This is because the three $s p$ hybridizations of carbon have an energy cost readily offset or exceeded by strong covalent bonding. The two recently discovered forms, $\mathrm{C}_{60}$ and most particularly carbon nanotubes, have aroused more interest than almost any other material. This is because of the special and unique properties associated with the structures and the prospect of tuning these properties for a purpose by nanoscale manipulation of structure. The desire to apply this knowledge within nanotechnology, where nanotubes, in particular, have been earmarked as key components, is a strong additional driving force.

The electronic structure of graphene, a single planar sheet of $s p^{2}$-bonded carbon atoms, is well understood and provides the basis for the elegant theoretical predictions of the electronic structure of single-walled carbon nanotubes (SWNT) made soon after their discovery [2]. These predictions are receiving backing through very recent experiments [3]. SWNT can be metallic or semiconducting, the latter with either a small or moderate gap, depending on the way in which their structures map onto the imaginary process of rolling up a graphene sheet, with refinements due to curvature effects [3]. However, this theoretical understanding is lost once intrinsic defects [4] are present in the graphene sheet. Defects matter for several reasons: Although they in general have high formation energies the synthesis routes used to make nanotubes do not have a high yield of defect-free specimens; defects undoubtedly alter the electronic structure and therefore chemical, optical, and other properties; and finally understanding scanning-tunneling microscopy images of $s p^{2}$-bonded carbon is difficult and there is unresolved debate over how defects manifest themselves [5]. A parallel concern is the chemical reactivity of carbon nanostructures and structure-property relationships therein. At the nanoscale electronic structure calculations become invaluable in understanding materials, and indeed the combination of theory and simulations has led the science of carbon nanotubes. Calculations on the electronic structure changes due to ring defects have appeared recently [6,7], as have some calculations on adsorption processes [8-10]. Our focus is on the interplay of defects and adsorption with the Stone-Wales (SW) defect as an example, using first-principles calculations to study the electronic structure of graphene with adsorbed hydrogen, with and without ring defects. The characteristic electronic gap state associated with hydrogen adsorbed on graphene is found to be very sensitive to the presence of such defects in the sheet, so that the gap state is a hallmark of hexagonal order in the sheet. We also attempt to explain the origin of the sizable gap opened by $\mathrm{H}$ adsorption. Our findings are related to $\mathrm{H}$ adsorbed on carbon nanotubes and to the subject of nanomagnetic carbon. The presence of defects has a strong influence on hydrogen chemical reactivity, shifting the adsorption energy from highly unfavorable in the perfect graphene case to virtually energy neutral over a SW defect in curved graphene. This is of relevance to the continuing debate over hydrogen storage in carbon nanotubes.

All calculations were performed using densityfunctional theory (DFT) implemented with plane waves and pseudopotentials as written into the CASTEP code [11]. In our model systems the separation between a graphene sheet and its periodic image in the surface normal direction was $c=15 \AA$; most results are taken from a 32-atom system where the calculated lattice parameters are $a=$ $b=9.84 \AA, \gamma=60^{\circ}$ (C-C bond length of $1.42 \AA$ ), although systems ranging in size from 16 to 98 atoms were employed, too. Because our systems contain hydrogen we used a gradient-corrected functional, specifically the revised Perdew-Becke-Ernzerhof [12] prescription. Vanderbilt ultrasoft pseudopotentials replaced the ion cores and a cutoff of $340 \mathrm{eV}$ was imposed on the planewave expansion, based on validation tests of graphene and small molecules. Graphene is a semimetal with zero band gap, but by analogy with nanotubes we expect that distortion of the graphene sheet may alter the electronic structure near $E_{F}$. Accordingly we have been cautious 
in choosing $\mathbf{k}$-point sampling meshes and energy-level smearing: The maximum spacing between $\mathbf{k}$ points was $0.03 \AA^{-1}$ and the smearing width was $0.1 \mathrm{eV}$ when at the ground state. Unless stated otherwise all structures are fully relaxed, meaning that in the final geometry no force exceeded $0.02 \mathrm{eV} / \AA$.

A necessary reference is the band structure of perfect graphene, which has been calculated and discussed many times [1]. For our purposes the most important feature is that the $\pi$ and $\pi^{*}$ bands touch at $K$.

Using a 32-atom supercell we calculate that the chemisorption of atomic hydrogen on graphene is endothermic by $1.53 \mathrm{eV}$ per $\mathrm{H}$ with respect to $\frac{1}{2} \mathrm{H}_{2}$. For the $\mathrm{H}_{2}$ energy we obtain $4.57 \mathrm{eV}$ [13]. To realize this adsorption in a real system would require the presence of $\mathrm{H}$ atoms or ions in the synthesis. As expected the adsorption induces some change towards $s p^{3}$ hybridization in the carbon atom to which bonding occurs, and this leads to some "tetragonalization." The $\mathrm{C}-\mathrm{H}$ bond length is $1.11 \AA$, typical of covalent bonding (cf. $1.09 \AA$ in methane). The most interesting result, however, is the electronic structure, shown in Fig. 1. A substantial gap of $1.25 \mathrm{eV}$ between the occupied and unoccupied graphene bands is opened up and two nondispersing states, the lower of which is singly occupied, lie in this gap [14]. The occupied gap state lies $0.45 \mathrm{eV}$ above the valence-band maximum. We return to the origin of the gap opening and the spin-polarized state shortly.

The presence of a Stone-Wales defect has a strong effect on the electronic structure of this system. This is illustrated in Fig. 2, which shows the band structure obtained when $\mathrm{H}$ is adsorbed on one of the $\mathrm{C}$ atoms in the "rotated" bond. The ground state is now metallic and spin paired. It can be argued [6] that the introduction of SW defects in graphene creates a substantial Fermi surface. Here this metallization effect clearly overcomes the gap opening caused by $\mathrm{H}$ adsorption. It might be thought that adsorption over the $\mathrm{C}$ atoms that form the $\mathrm{SW}$ defect is different from that over perfect graphene due to local differences in hybridization, but, in fact, this appears not
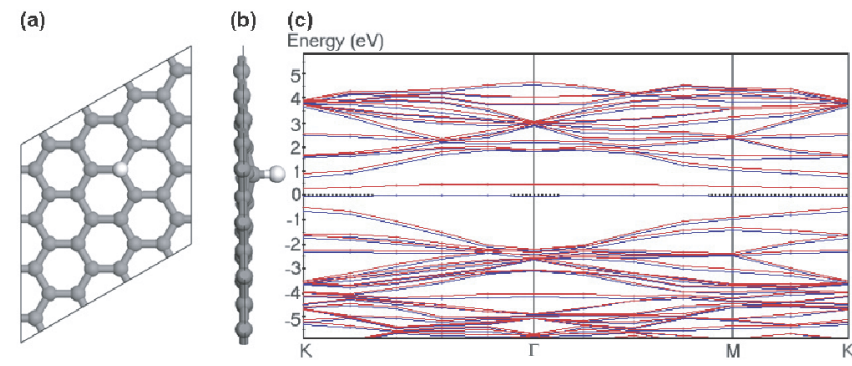

FIG. 1 (color online). Model system in plan view (a) and side view (b) and its band structure (c) for $\mathrm{H}$ (light sphere) adsorbed on a 32-atom graphene sheet. In (c) the path is through the Brillouin zone of the graphene unit cell. Spin-up and spin-down channels are represented by dark and light (blue and red online) lines, respectively. to be so. We repeated the calculations on much larger systems of $98 \mathrm{C}$ atoms, one with no defect and one containing a single $\mathrm{SW}$ defect. As before, $\mathrm{H}$ adsorbed on perfect graphene results in a spin-polarized gap state, but the spin is quenched when $\mathrm{H}$ is adsorbed either on the SW defect (on an atom in the rotated bond) or distant from the defect, i.e., on a graphene-like region of a sheet containing a SW defect. It appears that the metallization caused by the SW defect is responsible for quenching the spin. Put another way, the $p_{z}$ orbitals are no longer able to form the localized states that are required to maintain spin polarization since they are involved in extended metallic bands.

Making the plausible generalization from the SW defect to five- or seven-membered rings, an important conclusion to draw is that the spin-polarized state associated with $\mathrm{H}$ adsorption is a good indicator of the absence of such ring defects in graphene. The relationship between spin and defects may also matter in cases where the spin is the primary concern. One such case is magnetic nanographene [15], that is, a finite graphene sheet whose edges are passivated with hydrogen. It has been predicted that with the appropriate degree of edge hydrogenation a spinpolarized band is present with an accompanying magnetic moment. Ring defects induced in such a structure should have the ability to "switch off" the magnetism. We note in passing our results show that hydrogen need not be bound only at the edge of a graphene sheet for a gap state to arise.

It is interesting to consider why $\mathrm{H}$ adsorption opens such a substantial gap in the perfect graphene band structure. There are three main candidate mechanisms: First, the distortion of the flat graphene sheet may be expected to open a gap by analogy with curvature effects in nanotubes (see Ref. [3] and references therein); second, like any periodic potential (recall the periodic boundaries in use) the $\mathrm{H}^{+}$ionic core may open a gap; and third, the $\mathrm{C}-\mathrm{H}$ bonding orbital may itself induce band shifts. We have tackled this problem via a set of calculations. Taking the fully relaxed structure of a supercell of $32 \mathrm{C}$ atoms and adsorbed $\mathrm{H}$ we removed the neutral $\mathrm{H}$ atom and, keeping the $\mathrm{C}$ atoms fixed, recalculated the band structure. The



FIG. 2 (color online). As in Fig. 1 but with a Stone-Wales defect. The ground state is not found to be spin polarized in this case. 
result is a return to almost the perfect graphene band structure, with only a very small gap of $0.06 \mathrm{eV}$ at the $K$ point. This eliminates the curvature or distortion of the sheet as the origin of the large band gap. Next we took again the fully relaxed structure of the $32 \mathrm{C}$ atom supercell with adsorbed $\mathrm{H}$ and removed an electron, compensating the charge imbalance by adding a uniform background charge of $-e$. This mimics ionization of the system and enables us to gauge the effect of the perturbing potential of the $\mathrm{H}^{+}$ion. The calculated band structure, again with the ions held fixed, is qualitatively the same as that of the neutral system (Fig. 1) except that both gap states are unoccupied and degenerate [16]. The gap between occupied and unoccupied graphene bands is $1.39 \mathrm{eV}$, and the gap states lie $0.68 \mathrm{eV}$ above the valenceband maximum. Clearly the $\mathrm{H}^{+}$ion is responsible for opening a large gap; exchange splits the two gap states upon occupation of one of them. To complete our results, Fig. 3 shows the calculated density of states for six of the key situations discussed in the foregoing; this provides a concise summary of the electronic structure changes and their origins.

Can we relate our findings to carbon nanotubes? This must be done with caution since there are subtle effects on the electronic structure of SWNT due to their physical structure. The effect of introducing SW defects on SWNT

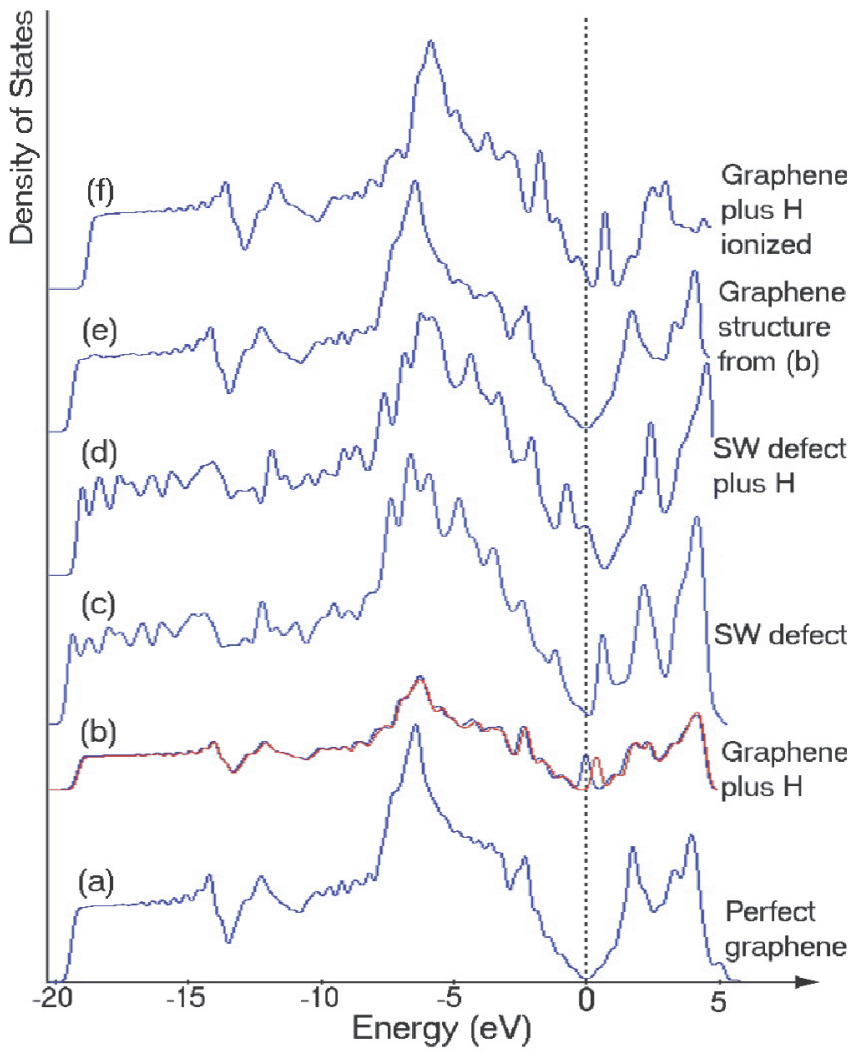

FIG. 3 (color online). Electronic density of states for perfect and imperfect graphene. On curve $b$ the spin-up and spin-down gap states lie at and above the Fermi level. electronic structure has been studied theoretically [6] and may be summarized as follows: The metallic armchair $(n, n)$ tubes see their density of states at $E_{F}$ increased; $(n, n+3 i)$ tubes, $i$ being an integer, which have small gaps $(\sim 0.1 \mathrm{eV})$ due to curvature-induced rehybridization, show an increase and then a decrease in band gap with defect concentration; and other $(n, m)$ tubes have their band gap closed by moderate concentrations of SW defects. We must now pose two questions: Does a gap state accompany $\mathrm{H}$ adsorption on SWNT, and is that state quenched by SW defects in the tube? Our preliminary calculations on $(3,3),(6,0)$, and $(7,0)$ SWNT show that in all cases a chemisorbed $\mathrm{H}$ atom opens a band gap and generates a gap state. Introduction of a SW defect into the $(3,3)$ and $(6,0)$ tubes, both cases corresponding to 1 in $72 \mathrm{C}-\mathrm{C}$ bonds rotated, quenches the spin; in the $(7,0)$ tube the gap state persists when the SW defect is incorporated ( 1 in $84 \mathrm{C}-\mathrm{C}$ bonds rotated). This latter result tallies with the defect-concentration dependence just discussed [6], since there metallization occurs somewhere between $1 / 84$ and $1 / 42$ rotated C-C bonds. We expect that a second SW defect in our $(7,0)$ tube would quench the spin.

Finally we report how a combination of a SW defect and high curvature greatly increases binding. Adsorption in the geometry shown in Fig. 4 is calculated to be endothermic by just $0.15 \mathrm{eV}$ per $\mathrm{H}_{2}$ molecule, in contrast with chemisorbed $\mathrm{H}_{2}$ on an equivalent-sized model of graphene $(2.01 \mathrm{eV})$. Both the curvature and the SW defect act to reduce the energy: Adsorption on a SW defect in planar graphene is endothermic by $0.67 \mathrm{eV}$, whereas on similarly modulated graphene with no defect it requires $0.47 \mathrm{eV}$. Note from Fig. 4 that the $2 \mathrm{H}$ atoms are adjacent and this, too, is important, as all of the many alternative arrangements we have tried yield higher system energies. The $\mathrm{C}-\mathrm{H}$ bond lengths are both $1.10 \AA$, the $\mathrm{C}-\mathrm{C}$ bond beneath the $\mathrm{H}$ atoms is stretched from 1.37 to $1.54 \AA$, and the separation of the $\mathrm{H}$ atoms is $2.27 \AA$. Several authors have examined $\mathrm{H}$ adsorption on a SW defect using periodic graphene models and DFT [8] or clusters and a "discrete variational method" [10]. Several studies have been directed at $\mathrm{H}$ adsorption on nanotubes, e.g., using self-consistent charge density-functional-based tight binding [17] and DFT [9,18]. These studies show that on small tubes chemisorption becomes exothermic and that

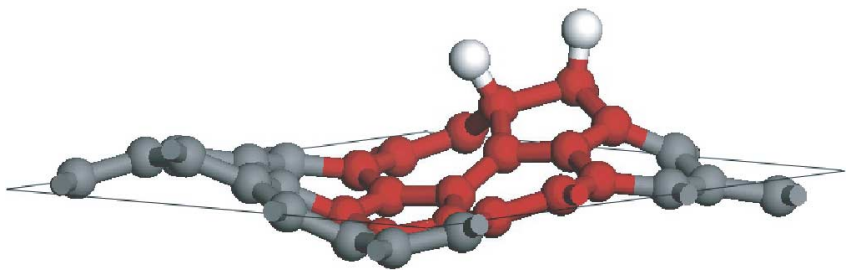

FIG. 4 (color online). Modulated graphene sheet containing a Stone-Wales defect upon which $\mathrm{H}_{2}$ is dissociated. The sheet is modulated to produce armchairlike curvature, and the maximum curvature is equivalent to that of a $(5,5)$ nanotube. 
increased curvature [18] leads to stronger binding. However, to the best of our knowledge, no study has incorporated the three ingredients that together appear to produce the most energetically favorable dissociated state, they being curvature, the SW defect, and adjacent adsorption sites. We have demonstrated this for graphene, and we suggest that chemisorption over a SW defect in a small nanotube will be more strongly exothermic than on the same tube with no defect. The barrier to dissociation is likely to be substantial, as, e.g., is that calculated for a $(5,5)$ nondefective nanotube [9].

In conclusion, we have shown how the adsorption of atomic hydrogen on graphene opens a substantial gap between the occupied and unoccupied graphene bands and is accompanied by a spin-polarized gap state. We suggest that it is the potential of the $\mathrm{H}^{+}$ionic core that causes the gap to open. A Stone-Wales defect anywhere in the graphene sheet down to a concentration of $1 / 147$ rotated $\mathrm{C}-\mathrm{C}$ bonds quenches the spin. We note that this provides a signal for the presence of ring defects in graphene, and we suggest that for certain nanotubes this is also true. This effect also provides a means to switch nanomagnetic graphite. Finally we have demonstrated that in graphene the combination of curvature and adjacent adsorption sites over a Stone-Wales defect increases binding for chemisorbed $\mathrm{H}_{2}$, and we suggest it should act similarly in nanotubes.

We acknowledge the EPSRC for supporting E. J. D. and for allocations of time on the CSAR and HPC(x) national facilities through the UKCP (GR/N02337/01) and Materials Chemistry (GR/N20607/01) consortia. We thank Johan Carlsson for fruitful discussions.

\footnotetext{
*Electronic address: p.lindan@kent.ac.uk
}

[1] R. Saito, G. Dresselhaus, and M. S. Dresselhaus, Physical Properties of Carbon Nanotubes (Imperial College Press, London, 1998).

[2] N. Hamada, S. ichi Sawada, and A. Oshiyama, Phys. Rev. Lett. 68, 1579 (1992).

[3] M. Ouyang, J.-L. Huang, and C. M. Lieber, Acc. Chem. Res. 35, 1018 (2002).

[4] By intrinsic we mean changes in bonding that disrupt the hexagonal structure. We do not consider vacancies, adatoms, etc.

[5] F. Atamny, O. Spillecke, and R. Schlögl, Phys. Chem. Chem. Phys. 1, 4113 (1999).

[6] V. H. Crespi, M. L. Cohen, and A. Rubio, Phys. Rev. Lett. 79, 2093 (1997).

[7] J.-C. Charlier, Acc. Chem. Res. 35, 1063 (2002).

[8] S. Letardi et al., Surf. Sci. 496, 33 (2002).

[9] J.S. Arellano et al., J. Chem. Phys. 117, 2281 (2002).

[10] L. G. Zhou and S. Q. Shi, Carbon 41, 613 (2003).

[11] M. D. Segall et al., J. Phys. Condens. Matter 14, 2717 (2002).

[12] B. Hammer, L. B. Hansen, and J. K. Nørskov, Phys. Rev. B 59, 7413 (1999).

[13] A zero-point correction of $0.26 \mathrm{eV}$ applies for molecular hydrogen; in considering adsorption we have neglected zero-point energies since the formation of two $\mathrm{CH}$ bonds largely offsets the loss of one $\mathrm{H}-\mathrm{H}$ bond.

[14] With a doubled supercell we have checked the difference between ferromagnetic and antiferromagnetic order, the former being favored by $0.08 \mathrm{eV}$.

[15] K. Kusakabe and M. Maruyama, Phys. Rev. B 67, 092406 (2003).

[16] This calculation gives a strong indication that the gap state would remain in the gap if self-interaction corrections were applied.

[17] S. M. Lee et al., J. Am. Chem. Soc. 123, 5059 (2001).

[18] O. Gülseren, T. Yildirim, and S. Ciraci, Phys. Rev. B 66, 121401 (2002). 